\title{
English Reading Comprehension as a Predictor for Academic Successes in First Year B. SC. Nursing Course in India
}

\author{
Shubhada Ponkshe, MSN,RN. \\ Vice-Principal/Associate Professor, Maharshi Karve Stree Shikshan Samstha's, Smt Bakul Tambat Institute of \\ Nursing Education.Karvenagar, Pune- 411052,Maharashtra, India.
}

\begin{abstract}
A research study is carried out to test the premise that poor ability of reading comprehension of study material in English affects academic performance. The sample used was seventy six fresh second year B.Sc. nursing students. The findings showed positive correlation between English reading comprehension ability and academic achievement obtained $\boldsymbol{r}$ values range from 0.48 to 0.65 are significantly greater than $p$-value .30 at $1 \%$ level of significance of a student implying that the students with better ability of English reading comprehension are expected to perform well in academics as far as nursing education is considered.
\end{abstract}

Keywords: Reading comprehension, Academic performance, Statistical correlation

\section{Introduction}

There are about 1652 languages belonging to five different language families in India. Out of such a vast number of languages, 87 languages are used in the print media, and 71 languages are used on radio. Eighth Schedule of the Indian Constitution recognizes 22 languages as official languages of the country; while the administration of the country is run in 13 different languages. Thus, multilingualism is constitutive of Indian diversity [1].

As far as foreign languages are considered, English is the language which has highest influence on people of India. This influence is due to the fact that Great Britain ruled over India almost for 150 years. Also, this rule was spread almost over the whole country. In spite of richness in diversity of languages in the country, there was no single language of Indian origin which could unite the whole country. Hence, English which had its influence in all fields became a natural choice for playing the role of a language of communication to people in this country. This choice of English as a common language was also dictated by the fact that English has been accepted as a language of communication almost all over the world.

In education system in India, English language is included as a second language in school education. However, for most of the students, medium of instruction during school education is the regional language and not English.

For higher education English language is the commonly used language as a medium of instruction. Indian nursing educational system is no exception to this reality. English is used as official language and also as medium of instruction for nursing courses at university level. Most of the available study material and literature in nursing is in English. As a result, nurses are required to have a certain level of ability to comprehend English in areas such as, reading text books and related literature; and communication at workplace.

Keeping in mind this importance of English, the controlling body namely, Indian Nursing Council (INC) has set an eligibility criterion that those students who are seeking admission to Basic B. Sc. Nursing course should have secured $50 \%$ marks in the group of subjects namely Physics, Chemistry, Biology with and English (PCBE) in the higher secondary science examination (HSSC). Along with this admission criterion, for making these nursing students more adaptable to nursing course and for enhancing the ability to comprehend spoken and written English required for effective communication in their professional work, INC has designed a 60 hours English course in the syllabus of first year Basic B.Sc. Nursing program.

Reading comprehension is an understanding of text that is read or the process of constructing meaning from a text. As comprehension takes place, words are decoded and associated with their meanings in the reader's memory, so that the meanings derived from one word, phrase, or sentence are not lost before the next is processed. Comprehension is the primary purpose of reading [2]. Comprehension becomes especially important to students in the later grade learning because it provides the foundation for further learning [3]. Success with nursing curriculum requires reading comprehension [4]. 
H.White, noted that to be successful in nursing courses, students must be able to read and comprehend a large volume of information which is required in nursing. Hence, reading comprehension is a crucial link in the process of cognitive learning and makes it a strong factor in educational and professional lives [5].

Being a nurse educator and evaluator for university examination, the researcher found that many students of first year basic B.Sc. Nursing course fail to perform well at University examination. The researcher felt that one of the reasons behind this failure may be poor ability of comprehending the read material which is always in English. This poor performance might possibly be due to the fact that these nursing students come from different states of India with multi-lingual and multi-cultural back ground and have studied English as a second language (ESL) during their schooling.

This researcher was curious to analyze whether the premise that "poor ability of reading comprehension of study material in English affects academic performance" is a reality or not. The researcher hence carried out a research to test this premise. Findings from this research are presented in this paper.

\section{Literature Review}

Nursing students whose English is second language face a variety of complex challenges during their nursing study period. Many study findings have reveled these challenges. A few study findings are presented below.

C .Fang,. et al. found that the barriers to learning English among nursing students were the lack of English competence, inadequate grammar, insufficient vocabulary, low listening comprehension, low speaking and pronunciation ability, low reading comprehension and the inability to write coherently [6].

Olson MA, did a critical review study of 25 articles. The purpose of this critical review of the literature is to identify barriers and discover bridges to ESL nursing student success. He identified that many ESL nursing students struggle in nursing school for a multitude of reasons. Language barriers were identified as the single most significant obstacle facing the ESL nursing students [7].

A nurse must be able to identify the client's psychosocial issues. As this is one of the areas where English proficiency can affect the performance, Hammett-Zelanko E., conducted comparative study to assess the psychosocial competency of English. The study was conducted between nurses having English as a second language (ESL) and non-ESL nurses groups. The study findings of her study adds to the body of nursing education literature on ESL nursing students by suggesting that there are specific groups of ESL nursing students that are at risk for lowered performance on the HESI (Health Education Specialist Incorporated) exam, specifically the HESI psychosocial needs questions. The HESI psychosocial needs questions relate to the student nurse's ability to appropriately respond to the requirements of those with emotional/psychosocial disorders/needs [8].

Sanner S, Astrid W. have studied the experiences of ESL students in a baccalaureate nursing program. For collection of data the researchers have conducted 3 interviews over a 6 month period. Authors noted that each participant shared instances where ESL may have contributed to his/her difficulty in academic progress. The authors also explored the reasons for course failure and have commented that description difficulties with reading and speech (including reluctance to speak in class) may have contributed to difficulty in academic progress [9].

Shakya A, Horsfall J M, interviewed 9 ESL nursing students to explore their experiences during one year of study. All participants experienced difficulty achieving the higher levels of verbal/written proficiency required to understand lectures, interact in tutorial groups and learn the more technical aspects of language in health terminology [10]

The findings of the qualitative study entitled "the lived experiences of students as English language learners in the nursing classroom", by JoAnn Mulready-Shick, suggest that the key to participants' academic progress involves additional academic learning time dedicated to studying the English language, along with learning the language of health care and nursing concepts [11].

In a paper entitled "Clinically speaking: A communication skills program for students from non-English speaking backgrounds", Miguel et al. , have reported that students having non-English speaking background experience difficulties in interacting with patients and staff during clinical practice, which places them at a high risk of failure. Authors further suggested that a specially designed clinically speaking program improved the 
experience of students from non-English speaking backgrounds during clinical placement and assisted the majority to successfully gain a satisfactory grade for their clinical placement [12].

Choi, wrote a review on the challenges faced by English-as-a-Second Language (ESL) nursing students. According to the author, nursing faculties need to address these challenges to meet the increasing diversity of the health care system. Further research into factors affecting the quality of nursing education for ESL students is necessary. Author further suggested a need for quantitative analysis to see if there exists a positive correlation between improved English language acquisition and academic success by ESL nursing students [13].

Esper, in her study has mentioned that the Assessment Technologies Institute (ATI) is a testing company that specializes in nationally standardized entrance testing for healthcare professions. ATI's Test of Essential Academic Skills (TEAS) measures math, English, and reading abilities as predictors of student success in healthcare programs including nursing. She determined the predictive ability of common ADN (Associate Degree Nursing) admission variables (TEAS scores in the areas of math, science and English; overall TEAS score; age; gender; and math, science, and English course grades) with regard to student success outcome variables. Using an exploratory, action research design, data from 120 freshman nursing students was examined to assess the relative contributions of each of the predictor variables on forecasting students' first semester success. The TEAS overall score correlated strongest with the outcome criteria, the English exam scores showed the highest correlation with the output variables [14].

Pritchard T L E, have investigated relationship between Computer Adaptive Placement Assessment and Support System (COMPASS) scores and successful completion of the Practical Nursing program. The COMPASS is an untimed, computerized test consisting of three distinct sections, English composition, mathematics, and reading. The reading portion of the COMPASS consists of various passages that the tester must read. The tester reads one passage, and then must answer a series of questions about the passage by selecting the answer from the multiple choices provided, before moving on to the next passage. The English portion of the COMPASS test also uses a multiple choice format, and presents a passage that the tester reads. The data analysis revealed a statistically significant relationship between the placement scores of completers versus non-completers on the reading subsection of the COMPASS [15].

Cook J D M, studied the relationship between reading comprehension skills as measured by the Nelson-Denny Reading Test and the Nurse Entrance Test and indices of academic success prior to admission for students in a Bachelor of Science in Nursing (BSN) program with student's success in the first semester of nursing coursework. Results of this study indicated that reading comprehension, as measured by the Nelson-Denny Reading Test, was better in identifying student risk for academic failure [16].

From the review it is evident that ample work has been done overseas in various educational faculties. The review has also established that English reading ability is an important variable which has impact on academic achievement, and for professional progress. However, no such research work is published from Indian nursing field, hence this researcher under took a study to examine whether there is a co-relation between English reading comprehension ability and academic achievement amongst first year basic B.Sc. Nursing students from India.

\section{Objectives}

\section{The objectives of the study were to}

- assess the scores of English reading comprehension ability test among nursing students.

- assess the correlation between scores obtained by concerned student in the university examination of the first year basic B.Sc. nursing course and scores of English reading comprehension ability test.

-assess the correlation between scores obtained by concerned student in the scores in Physics, Chemistry, Biology with and English (PCBE) in the higher secondary science examination and scores of English reading comprehension ability test.

\section{IV.1 Design and sampling}

\section{Methods}

An explorative survey design was used in this study. A non-probability convenient sampling technique was used. The participants were all fresh second year basic B.Sc. nursing students who have done their schooling in vernacular medium and have studied English as their second language and who have just finished their English course of first year basic B.Sc. nursing. The sample size was 76 . 


\section{IV.2 Instrument}

Owing to non-availability of any appropriate standardized test to measure English reading comprehension ability of nursing students from India; the researcher decided to construct this test herself. Hence the researcher developed a test comprising of multiple choice questions which are grouped in to vocabulary testing questions and comprehension testing questions. The items for the test were chosen from the pool of already available tests or constructed where required. All questions in the test were recognition type questions. These test questions were divided into five sections namely reading for general significance, reading to note details, synonyms, antonyms and recognition of words in context.

The test was validated for its content by language experts. Suggestions given by the experts were taken into consideration and the test was modified accordingly. The reliability of the test was also found out by using rational equivalence method. The test was found to be adequately reliable with the reliability quotient of .82 .

The marks obtained in PCBE group (PCBE group scores) at higher secondary school level examination and the marks obtained in the first year basic B.Sc. nursing course university examination were obtained from the college record.

\section{IV.3 Data collection procedure}

The data collection was started after the approval of the institution's authority, research committee and obtaining informed consent from the participants. English reading comprehension ability scores of each participant were obtained by administrating English reading comprehension ability test. The marks obtained in PCBE group (PCBE group scores) at higher secondary school level examination and the marks obtained in the first year basic B.Sc. nursing course university examination were obtained from the college record.

\section{Results And Discussion}

The first year basic B.Sc. nursing course university examination consists of 5 theory papers namely anatomy \& physiology, nutrition \& biochemistry, nursing foundation, psychology, and microbiology. Each paper is for 75 marks. For the purpose of analysis, marks obtained by a student in each paper, as well as aggregate marks were converted to percentage scores. The marks obtained in English reading comprehension ability test were also converted to percentage scores.

While assessing correlation between English reading comprehension ability and performance in university examination in a particular paper or aggregate performance; scatter diagrams were plotted. In the scatter diagrams, the students get represented as different points with their scores in English reading comprehension test as $\mathrm{X}$ coordinates and their scores in a particular paper or aggregate scores as $\mathrm{Y}$ coordinates.

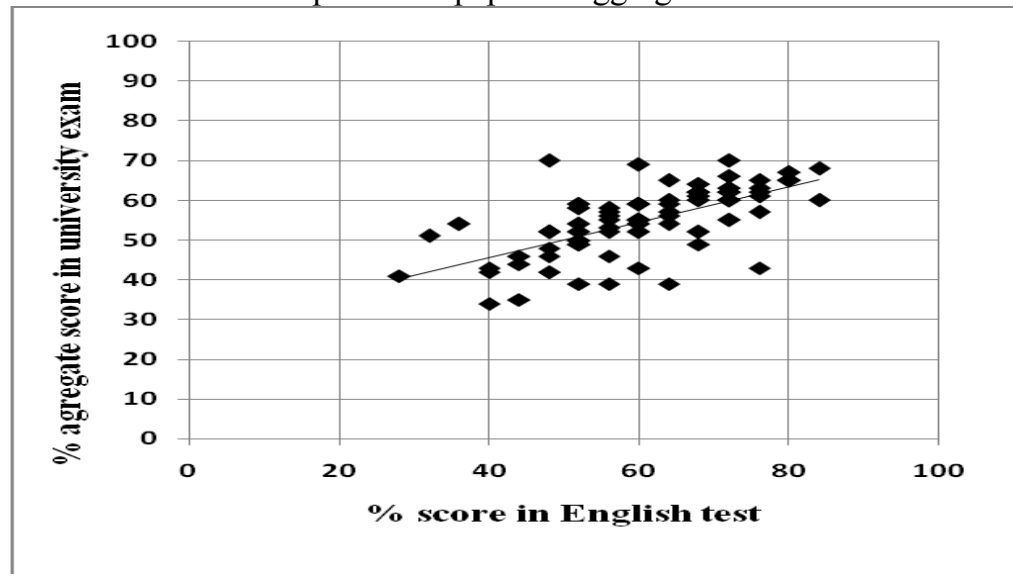

Figure 1. Scatter diagram representing correlation between aggregate performance in first year basic B.Sc. nursing course university examination and English reading comprehension ability.

Fig- 1 is a representative scatter diagram representing correlation between aggregate scores in first year basic B.Sc. nursing course university examination and English reading comprehension ability test scores. This scatter diagram clearly reveals that the points scattered are densely placed near the line of regression. This obviously indicates that there is a positive correlation between English reading comprehension ability test scores and aggregate performance in first year basic B.Sc. nursing course university examination.

Similar scatter diagrams were plotted to examine, the correlation between English reading comprehension ability test and performance in university examination in a particular paper. Though the diagrams are not 
included here, the researcher has observed that there is a positive correlation between English reading comprehension ability and performance in all individual theory papers of first year basic B.Sc. nursing course university examination.

To quantify the correlation between English reading comprehension ability and performance in university examination in a particular paper or aggregate performance, coefficients of correlation were calculated using Pearson product moment method. Windows 2007 excel analysis tool pack was used to calculate these coefficients. These coefficients are tabulated in Table 1.

Table 1

Coefficients of Correlation Between English Reading Comprehension Ability and Aggregate Performance as well as Performance in a Particular Theory Paper of First Year basic B.Sc. nursing course university examination.

\begin{tabular}{lc}
\hline \multicolumn{1}{c}{ Variables } & $\begin{array}{c}\text { Coefficient of } \\
\text { correlation (r) }\end{array}$ \\
\hline $\begin{array}{l}\text { Aggregate performance in first year basic } \\
\text { B.Sc. nursing course university examination }\end{array}$ & 0.65 \\
\hline $\begin{array}{l}\text { Performance in theory paper of anatomy \& physiology in first year } \\
\text { basic B.Sc. nursing course university examination }\end{array}$ & 0.55 \\
\cline { 1 - 1 } $\begin{array}{l}\text { Performance in theory paper of nutrition and biochemistry in first year } \\
\text { basic B.Sc. nursing course university examination }\end{array}$ & 0.48 \\
\hline $\begin{array}{l}\text { Performance in theory paper of fundamentals of nursing in first year } \\
\text { basic B.Sc. nursing course university examination }\end{array}$ & 0.51 \\
\hline $\begin{array}{l}\text { Performance in theory paper of psychology in first year } \\
\text { basic B.Sc. nursing course university examination }\end{array}$ & 0.52 \\
\hline $\begin{array}{l}\text { Performance in theory paper of microbiology in first year } \\
\text { basic B.Sc. nursing course university examination }\end{array}$ & 0.61 \\
\hline
\end{tabular}

The values of coefficient of correlation range between minimum value of 0.48 in the case of performance in the theory paper of nutrition and biochemistry and maximum value of 0.65 in the case of aggregate performance in first year basic B.Sc. nursing course university examination. All these values are indicative of sound positive correlation of academic performance in university examination with the English reading comprehension ability. Now, there may be chances that the population $r$ is 0 that is there is no correlation in the two variables under study in population and positive correlation found in case of sample might have arisen from fluctuations of sampling alone. Hence the significance of obtained $r$ was tested against the hypothesis that the population $r$ is 0 . The researcher found that all the $r$ values obtained (which range from 0.48 to 0.65 ) are significantly greater than $p$-value .30 for 74 (76-2) degrees of freedom at $1 \%$ level of significance. This clearly suggests that obtained values are highly significant to conclude that the obtained results are true for population.

The research findings have revealed that English reading comprehension ability of student has positive correlation with their academic achievement in university examination. Hence it can be concluded that the students with better ability of English reading comprehension are expected to perform well in academics as far as nursing education is considered.

Similar finding were reported by JDM. Cook studied the relationship between reading comprehension skills as measured by the Nelson-Denny Reading Test and the Nurse Entrance Test and indices of academic success prior to admission for students in a Bachelor of Science in Nursing (BSN) program with student's success in the first semester of nursing coursework. Results of this study indicated that reading comprehension, as measured by the Nelson-Denny Reading Test, was better in identifying student risk for academic failure [15].

Abdur-Rahman et al. have explored the correlation between the Nurse Entrance Test (NET) and first-year nursing program success. He used the Pearson $r$ correlation coefficient to determine relationships among the NET scores and nursing grades. He found a statistically significant relationship between NET reading comprehension and first semester nursing grades [17]. Czubatyj in their study found a co-relation between composite scores and in particular scientific reading comprehension and the graduation success rate [18]. The findings of the present study are hence in very good agreement with the findings of similar research studies carried out by many other researchers. 


\section{Conclusion}

In the present study, correlation between English reading comprehension ability test scores and aggregate scores in first year basic B.Sc. nursing course university examination was found to be much higher at 0.65 . English is taught as a subject in the first year of basic B.Sc. nursing programme and medium of instruction is also English. The study finding indicates that nursing students might be finding it difficult to comprehend the basic sciences taught in the first year nursing programme.

The researcher feels that students would also need to be assessed for their reading comprehension and understanding of the English language. When a student is unable to read a question correctly and understand what needs to be extracted from it, then the likelihood of success at university examination will decrease. If English reading comprehension ability is assessed at the entry level it may help faculty and administrators to plan additional training programme for those with poor abilities. As can be seen from the literature, many nursing institutes in other countries have found that such English language support programmes are quite effective. For instance, Crawford T, Candlin S. have discussed mixed results reported from a number of English language support programmes [19]. Hsiao-Yun et al. have carried out quasi experimental study to assess the impact of simulation-based learning on students' English for Nursing Purposes (ENP) reading proficiency. The findings indicated that the intervention group had significantly higher mean scores in ENP reading proficiency compared to the control group. Also, the intervention students showed more improvement in their English reading, both from quantitative and qualitative findings.[20] The researchers Ooms et al. have carried out a study into the use and perceived effectiveness of the support services offered to nursing students at two universities; where they found that nursing students at both universities appear to value the support they receive to enable them to succeed in their nursing programmes and perceive the support services to be accessible and available to many[21]. Hence the researcher of present study has decided to implement English language support system at her Institute.

\section{References}

[1] K. Kapur, Language Education in India: Dialoging English and Vernacular Languages and Literatures. Sino-US English Teaching, 8(5), 2011, 307-321.

[2] J. Kruidenier, Research-based principles for adult basic education reading instruction (USA, The National Institute for Literacy, 2002).

[3] A.P.Sweet, C. E. Snow, (Ed.), Rethinking reading comprehension,(New York, NY: Guilford Press. London. 2003).

[4] P.J. Worrell, Metacognition: Implications for instruction in nursing education, Journal of Nursing Education, 29 (4), $1990,170-175$.

[5] H. L. White, Nursing instructors must also teach reading and study skills. Reading Improvement, 41(1), 2004, 38-50.

[6] Y. Fang, C.Tsai, C. Huang, Needs and barriers in ESP education: Exploring the preference in ESL nursing students, Proc. International Symposium on ESP and Its Application in Nursing \& Medical English education, Taiwan. 2011.

[7] M.A. Olson, English-as-a-Second Language (ESL) nursing student success: a critical review of the literature, Journal of Cultural Diversity, 19(1), 2012, 26-32.

[8] E.Hammett-Zelanko, Psychosocial Competency of the English as a Second Language Nursing Student: A Comparative Study, doctoral diss.,Texas Woman's University, 2007. http://www.proquest.com.

[9] S .Sanner, W. Astrid, The experiences of students with English as a second language in a baccalaureate nursing program, Nurse Education Today, 28(7), 2008, 807-813.

[10] A .Shakya, J.M. Horsfall, ESL undergraduate nursing students in Australia: Some experiences. Nursing \& Health Science, 2(3), 2000, 163-171.

[11] J. Mulready-Shick, The lived experiences of students as English language learners in the nursing classroom: A critical hermeneutic inquiry, doctoral diss., University of Massachusetts, Boston; 2008. http://www.proquest.com

[12] C. S. Miguel, F .Rogan, K. Kilstoff, D. Brown, Clinically speaking: A communication skills program for students from non-English speaking backgrounds. Nurse Education in Practice, 6(5), 2006, 268-274.

[13] L.Choi Literature review: issues surrounding education of English-as-a second language (ESL) nursing students, Journal of Transcultural Nursing, 16 (3), 2005, 263-268.

[14] L. L.Esper, Admission Variables as a Predictor of First Semester Student Success: A Quantitative Investigation of an Associate Degree in Nursing Program, doctoral diss., College of Education, University of Hartford, 2009. http://www.proquest.com

[15] T L E. Pritchard, COMPASS and Prerequisite Course Scores as Predictors of Success in Practical Nursing School, doctoral diss., Walden University,2010. http://www.proquest.com.

[16] J D M. Cook, The relationship between reading comprehension skill assessment methods and academic success for first semester students in a selected bachelor of science in nursing program in Texas, doctoral diss,. Texas A\&M University, 2006. http://www.proquest.com.

[17] V.Abdur-Rahman, P.L. Femea , C. Gaines , The nurse entrance test (NET): An early predictor of success,Association of Black Nursing Faculty Journal, 5(1), 1994, 10-14.

[18] A.M.Czubatyj,A correlational study on the Nurse Entrance Test as a predictor of success in an associate degree of nursing program, doctoral diss, Capella University,2010.http://www.proquest.com.

[19] T. Crawford, S.Candlin, A literature review of the language needs of nursing students who have English as a second language and the effectiveness of English language support programmes, Nurse Education in Practice, 13 (3), 2013,181-185.

[20 ]Hsiao-Y, C. Annie, C. Luke, S. Betty, The impact of simulation-based learning on students' English for Nursing Purposes (ENP) reading proficiency: A quasi-experimental study, Nurse Education Today, 33(6), 2012, 584-589.

[21] A. Ooms, S. Fergy, D. Maran, L.Burke, K. Sheehy,Providing learning support to nursing students: A study of two universities, Nurse Education in Practice. 13 (2), 2013, 89-95. 